\title{
INPRO Activities on Development of Advanced Tools to Support Judgment Aggregation for Comparative Evaluation of Nuclear Energy Systems
}

\author{
V. Kuznetsov, ${ }^{1}$ G. Fesenko, ${ }^{1}$ A. Andrianov, ${ }^{2}$ and I. Kuptsov $^{2}$ \\ ${ }^{1}$ International Atomic Energy Agency, Wagramer Straße 5, P.O. Box 100, 1400 Vienna, Austria \\ ${ }^{2}$ National Research Nuclear University MEPhI (Moscow Engineering Physics Institute), Kashirskoe Shosse 31, \\ Moscow 115409, Russia
}

Correspondence should be addressed to A. Andrianov; andreyandrianov@yandex.ru

Received 5 September 2014; Revised 29 October 2014; Accepted 30 October 2014

Academic Editor: Giorgio Galassi

Copyright @ 2015 V. Kuznetsov et al. This is an open access article distributed under the Creative Commons Attribution License, which permits unrestricted use, distribution, and reproduction in any medium, provided the original work is properly cited.

\begin{abstract}
This paper presents first results of the INPRO Collaborative Project on Key Indicators for Innovative Nuclear Energy Systems, which has the objective to develop guidance and tools for comparative evaluation of the status, prospects, benefits, and risks associated with development of innovative nuclear technologies for a more distant future. Presented results illustrate expedience of application of the multicriteria decision analysis methods, which are able to provide the added value to comparative assessment of nuclear energy systems. First, the paper presents a short review of the multicriteria decision analysis methods appropriate to support judgment aggregation within comparative evaluations of nuclear energy systems based on key indicators and highlights the methodology to perform such assessments. Second, a set of key indicators elaborated in the INPRO Collaborative Project on Global Architecture of Innovative Nuclear Energy Systems Based on Thermal and Fast Reactors Including a Closed Fuel Cycle (GAINS) were evaluated for comparative evaluation of nuclear energy system evolution scenarios. Third, a numerical example is presented of application of the selected key indicators, methods, and tools for judgment aggregation in comparative assessment of the GAINS nuclear energy systems.
\end{abstract}

\section{Introduction}

The International Project on Innovative Nuclear Reactors and Fuel Cycles (INPRO) [1] was established in 2000 with the goal of ensuring a sustainable nuclear energy supply to help meet 21st century global energy needs. INPRO's activities are centred on the key concepts of global nuclear energy sustainability and the development of long-range nuclear energy strategies, so that nuclear energy is and remains available to meet national energy needs in interested IAEA Member States.

Collaborative Project on Global Architecture of Innovative Nuclear Energy Systems Based on Thermal and Fast reactors Including a Closed Fuel Cycle (GAINS) [2] developed an analytical framework for assessing nuclear energy evolution scenarios. The analytical framework (hereafter, the GAINS framework) is a part of the integrated services provided by IAEA to Member States considering initial development or expansion of their nuclear energy programmes.

Innovative technologies to support future sustainable nuclear energy systems are being analyzed/developed in many countries worldwide. With multiplicity of such developments the need periodically arises in Member States to evaluate the status, prospects, benefits, and risks associated with development of particular technologies as compared to others, in order to prioritize/adjust the allotment of financing and other resources within national programmes on innovative nuclear technology development. The INPRO Collaborative Project on Key Indicators for Innovative Nuclear Energy Systems (KIND) has the objective to develop guidance and tools for comparative evaluation of the status, prospects, benefits, and risks associated with development of innovative 
nuclear technologies for a more distant future. To achieve this objective the project is

(i) developing a limited number of Key Indicators (KI) to reflect the status, prospects, benefits, and risks associated with innovative technologies and nuclear energy systems (NESs) on their basis (Track 1);

(ii) adapting and verifying advanced methods of expert judgment aggregation within the assessments involving quantifiable data and expert opinions, to enable effective comparative evaluation of innovative NES based on the defined set of KI (Track 2).

The problem of evaluation, comparative analysis, assessment, and optimization of NES is a multicriterial one. The criteria characterizing different aspects associated with resource consumption, economics, proliferation risks, and waste management strategies are conflicting by nature. This means that improving the value of one criterion may lead to a decrease in the values of other criteria $[3,4]$.

Given a wide range of problems having multicriterial character in the area of NES assessment and different possible levels of assessment (e.g., complete NES level, a particular nuclear fuel cycle (NFC) level or reactor technology level, etc.), the multicriteria decision making (MCDM) technique is more suitable and recommended to be applied for comparative analysis and assessment. Implementation of the MCDM methods building upon the INPRO methodology and the GAINS framework may provide useful new information for ranking of the NES options based on a set of KI using a set of preferences.

In the framework of the Track 2 of the KIND project it is expedient to subdivide the problem into parts and move according to the logic "from simple to complicated." Such a consistent approach will simplify the analysis of the problems providing a clear understanding of which results can be obtained in the simplest assumptions and then sequentially to complicate the problems and methods being used. Such an approach provides an opportunity to analyze the use of more sophisticated MCDM methods in comparison with the simpler ones and to justify one most appropriate method.

Special attention in realization of the activities of Track 2 should be given to outlining the boundaries of possible application of the MCDM tools and to formulating general recommendations for their application in multicriteria assessment of NES or scenario effectiveness, without a reference to specific methods, key indicator sets, objects of evaluation, and so forth. At the same time, these activities should include elaboration of specific examples (case studies or numerical examples) showing what could be achieved with the application of the MCDM tools in this problem area (preferably, on the basis of well-known studies to clearly demonstrate the added value resulting from application of the MCDM tools).

The first results illustrating the expedience of application of some well-known multicriteria decision analysis methods for comparative assessment of NESs analyzed in the framework of the GAINS project are presented in this paper. The paper also provides recommendations regarding applicability of the corresponding methods for the KIND objectives showing added value that may be obtained by implementation of the MCDM tools for NESs and scenario comparative assessment. Numerical examples are provided for a set of key indicators for comparative evaluation of NES evolution scenarios elaborated in the INPRO Collaborative Project on Global Architecture of Innovative Nuclear Energy Systems Based on Thermal and Fast Reactors Including a Closed Fuel Cycle. This does not limit the applicability of the derived conclusions to other problems (other key indicator sets), for example, those addressed within Track 1 of the KIND project.

\section{Multiple-Criteria Decision Making Framework}

Multiple-criteria decision making techniques are a tool aimed at supporting decision makers who are faced with making numerous and conflicting assessments and intend to highlight conflicts and find compromises in the decision making process [5, 6]. Multicriteria Decision Analysis (MCDA) and Multiobjective Decision Making (MODM) are the main components of MCDM. There are different classifications of MCDM problems and methods. The major distinction between MCDM problems is based on whether the solutions are defined explicitly or implicitly.

The MCDA problems consist of a finite number of alternatives, explicitly known in the beginning of the decision support process. Each alternative is represented by its performance in multiple criteria. The problem may be defined as finding the best alternative for a decision maker, or finding a set of acceptable trade-off alternatives. In the MODM problems, the alternatives are not explicitly known. An alternative (solution) can be found by solving a mathematical problem. The number of alternatives is either infinite or not countable (when some variables are continuous) or typically very large, if countable (when all variables are discrete).

MCDA methods are the most adequate for the KIND approach and objective. The MCDA methods provide an opportunity not only to quantitatively evaluate the most vulnerable areas of the considered NES, but also to identify the cost-effective risk mitigation measures and assess the effect of the implementation of these measures to reduce risks associated with the corresponding vulnerabilities.

2.1. MCDA Application to Comparative Assessment of NESs. The decision support process starts with identification of the decision maker, the group of experts, and the stakeholders (persons interested in a certain decision) and further goes through the following steps: problem formulation, formulation of alternatives, criteria identification, criteria assessment, selection of MCDA method, uncertainty and sensitivity analysis, final conclusions, and recommendations.

The "problem formulation" step should provide a clear statement of the problem, reflecting its current state and vision of the preferred result. An agreement should be reached regarding the problem formulation to avoid possible further misunderstandings in the decision support process. At this step it is necessary to specify clearly the requirements, conditions, and restrictions that must be satisfied by any possible solution of the problem. The goal formulation is 
another important component of the problem formulation step specifying directions to find the solution.

The "formulation of alternatives" step should provide an identification of possible ways to achieve the goals (a solution, the alternative, etc.). Each alternative must meet all identified requirements, conditions, and restrictions. The number of alternatives may be finite or infinite.

The "criteria identification" step should provide a formulation of quantitative parameters concretizing the goals which may be subsequently measured for each alternative. Each goal must match one or more criteria. The criteria can be either qualitative or quantitative and, in some MCDA methods, satisfy a certain set of requirements (not to be redundant, to be independent, etc.).

The "criteria assessment" step should provide an evaluation of alternatives according to the proposed criteria and development of the so-called performance table on this base. This information is required for each MCDA method, as input data. The assessments may be expressed quantitatively or qualitatively; they may be objective or subjective.

The "selection of MCDA method" step should provide a selection of the most suitable method which depends on the nature of the problem being solved. On the basis of the method chosen it is then necessary to make an assessment of the alternatives.

The "uncertainty and sensitivity analysis" step should provide justification for the resulting solutions, whichever method was used or whatever alternative was chosen. Such analysis helps to identify stability and robustness of the solution under given uncertainties in the considered criteria values and preferences, as well as possible ways to restructure the problem, including revision of the considered criteria, alternatives, and methods used.

The "final conclusions and recommendations" step provides a formulation of recommendations to decision makers regarding the more suitable trade-off solutions identified during implementation of the decision support process. If an outcome does not satisfy the decision maker, analysts should return to the previous steps.

The properly organized on the basis of the MCDA paradigm studies represent a process not only formally operating with a set of mathematical methods and various analytical tools, but also leading to a comprehensive understanding of the problem and its elaboration. Multicriteria decision analysis does not provide a "right solution"; in this regard it would be correct to talk about a compromise or a trade-off solution, paying special attention to analysis of the solution stability to the various methods used and their model parameters.

2.2. MCDA Methods Applied in the Study. The MCDA methods provide for analysis of the predefined set of alternatives. MCDA is applied to the following problem: given a set of $M$ alternatives and $N$ criteria for their assessment, one shall assume that each of the alternatives has been evaluated by each of the criteria either by experts or through objective calculations. It is then necessary to derive a rule from the experts' preferences, which will allow ranking the alternatives according to their values and identifying the best among them.
A large number of MCDA techniques have been developed to deal with different kinds of problems. The assessments presented in this study were made using the following well-known and widely used MCDA methods: MAVT (Multiattribute Value Theory), MAUT (Multiattribute Utility Theory), TOPSIS (Technique for Order Preference by Similarity to the Ideal Solution), PROMETHEE (Preference Ranking Organization Method for Enrichment Evaluations), AHP (Analytic Hierarchy Process), and simple scoring model (SSM) [7-11]. In order to verify the calculation results, the various software tools were applied. In the framework of the classical deterministic MCDA methods, for example, these used in this study MAVT, PROMETHEE, TOPSIS, AHP, and SSM, the criteria values and weights are real undistributed (i.e., nonrandom) numbers. In such methods, uncertainties are examined by means of the sensitivity analysis, generally by applying it to changes in the values of weights.

For practical purposes, KI values for an alternative are determined ambiguously due to various uncertainties. In the presented study, uncertainties may be taken into account only within the MAUT method. Criteria values and a multiattribute utility function can be represented by random variables. The MAUT alternative ranking procedure is based on calculation of an expected utility function (mathematical expectation of a multiattribute utility function). Despite widespread use of the expected utility function treated as a universal tool to justify the preferences, its application to the choice of alternative ranking is not widely accepted.

\subsection{Assumptions Made in the Study}

2.3.1. The Multiattribute Value Theory (MAVT). Linear functions were chosen as the single-attribute value functions for the considered KI. The reasonableness of this assumption was confirmed by sensitivity analysis of the type of singleattribute value functions. Because the particular problem considered was to minimize the values of all of the KI, monotonically decreasing linear functions were selected as the single-attribute value functions.

2.3.2. The Multiattribute Utility Theory (MAUT). MAUT is a theory closely related to MAVT, which is based upon the expected utility theory. MAUT extends MAVT in using probabilities and expectations to deal with uncertainties. A criterion value uncertainty is represented in MAUT by a random variable with the probability density function. The overall utility for the alternatives can be considered in this case a random variable. The alternative ranking within MAUT is based on the comparison of expected utilities: one alternative exceeds the other if the mathematical expectation of a utility function for the first alternative is greater than that of the other.

Single-attribute value function (in the MAVT method) and single-attribute utility function (in the MAUT method) transform the value of KI from a criterion "natural" scale to the score range (from 0 to 1 ). These scores are used in further calculations. A single attribute value function expresses an expert's preference regarding different levels of an attribute 
under certainty, whereas utility theory extends the method to use probabilities and expectations to deal with uncertainty. Among all other MCDA methods the MAVT and MAUT methods have been applied to a wide range of decision making problems in the area of multicriteria comparative assessment of nuclear reactors, related NFCs and NESs [1219].

2.3.3. The Technique for Order Preference by Similarity to the Ideal Solution (TOPSIS). TOPSIS is based on a concept that the chosen alternative should have the shortest distance from the most desirable (ideal, or positive ideal) solution and the longest distance from the less desirable (anti-ideal, or negative ideal) solution. Ideal solution is a solution which has the best level for all indicators considered. Negative ideal solution is a solution which has the worst indicator values. TOPSIS selects the solution that is the closest to the ideal solution and farthest from negative ideal solution.

\subsubsection{The Preference Ranking Organization Method for} Enrichment Evaluations (PROMETHEE). The PROMETHEE method belongs to the so-called outranking methods which imply forming an ordered relation of a given set of alternatives. Outranking methods are based on a pairwise comparison of alternatives for each criterion under consideration, with subsequent integration of the obtained preferences according to a chosen algorithm. In the PROMETHEE method it is required to choose preference function defined in the range from 0 to 1 , with specified indifference and preference thresholds.

2.3.5. The Analytic Hierarchy Process (AHP). To obtain estimates by the AHP method, matrixes of pairwise comparisons were filled in accordance with the AHP algorithm based on weights and the performance table specified in the AHP pairwise comparison scale.

Additionally, the simple scoring model (SSM) was used for comparison. Results were visualized by means of color code, heat map techniques, and scatter graphs of highlevel objectives values to provide better interpretation and illustration of ranking results and conclusions.

The approach implemented in this study consisting of using several different MCDA methods may facilitate thorough understanding, recognizing and analyzing the problem, providing an additional sensitivity analysis of the obtained ranking results to the methods used that increase the confidence level to the study. Application of a wide landscape of different methods may have a significant influence on subsequent decision making and help a decision maker more thoroughly understand and analyze the problem, achieving consistency in judgments and estimates, as well as necessitates examining the stability and robustness of the ranking results to different assumptions. Although the ranks of alternatives may vary for different MCDA methods, an analysis of the problem by different methods may play a significant role in an interactive process of understanding the problem and identifying its main features and it may demonstrate that different methods may provide noncontradictory results.

\section{Multicriteria Comparison of the GAINS Nuclear Energy System Evolution Scenarios}

3.1. The GAINS Analytical Framework. The NES evolution scenarios obtained in the GAINS collaborative project reflected in the IAEA Nuclear Energy Series NP-T-1.14 [2] were chosen for multicriteria comparison by abovementioned well-known deterministic MCDA methods to demonstrate the added value to the comparative analysis which is provided by the application of the MCDA tools. The GAINS analytical framework presents a tool for evaluation of the NES evolution scenario key parameters on the entire system level, but without practical recommendations on the application of this information to formulate integrated expert judgments about preferable NES evolution scenarios and their sustainability potential. The GAINS framework was understood as a common methodological approach based on the same assumptions and boundary conditions with following major circumstances:

(i) long-term nuclear energy demand scenarios based on the IAEA Member States' high and low estimations on nuclear power deployment until 2030 and expected trends until 2050 and on forecasts of competent international energy organizations;

(ii) heterogeneous world model comprised of groups of nonpersonified nongeographical countries with different policy regarding NFC back-end;

(iii) architectures of NES;

(iv) metrics and tools for the assessment of dynamic INES evolution scenarios regarding sustainability, including a set of key indicators and evaluation parameters;

(v) an internationally verified database with characteristics of existing and advanced nuclear reactors and associated NFC needed for material flow analysis, extending the previously developed by the IAEA databases and taking into account preferences of different countries;

(vi) selected results of sample analysis for scenarios involving transition from the present fleets of nuclear reactors and NFC to future sustainable NES architectures involving innovative technological solutions.

A variety of the indicators were proposed in the GAINS project which may be used to assess the considered NES evolution scenarios efficiency. At the same time, there is a lack of practical recommendations on the application of such information to formulate integrated expert judgments regarding preferable NESs and their sustainability potential. In this situation the MCDA methods will represent a tool for applying the state-of-the-art approach to sorting, ranking, and multicriteria quantitative comparative assessment of the considered NES evolution scenarios efficiency that provides an opportunity to make definite judgments about the preferred NESs.

3.2. Nuclear Energy System Evolution Scenarios. Among more than 55 NES evolution scenarios considered in the framework 
TABLE 1: Considered nuclear energy system evolution scenarios.

\begin{tabular}{|c|c|c|c|}
\hline$N$ & Nuclear energy system scenario & $\begin{array}{c}\text { Scenario denotation } \\
(\text { reactor technologies mix })^{1}\end{array}$ & Color code \\
\hline 1 & BAU & L1H1 & \\
\hline 2 & $\mathrm{BAU}+$ & L1L2H1 & \\
\hline 3 & BAU+, FR "break-even" & L1L2H1F1 & \\
\hline 4 & BAU+, FR “medium-BR”, medium-burn-up & L1L2H1F2 & \\
\hline 5 & BAU+, FR “medium-BR”, high-burn-up & L1L2H1F3 & \\
\hline 6 & BAU+, FR "break-even" and ADS & L1L2H1F1A1 & \\
\hline 7 & BAU+, FR "medium-BR", medium-burn-up and ADS & L1L2H1F2A1 & \\
\hline 8 & BAU+, FR “medium-BR”, high-burn-up and ADS & L1L2H1F3A1 & \\
\hline 9 & BAU+, FR "break-even" and MSR & L1L2H1F1M1 & \\
\hline 10 & BAU+, FR “medium-BR", medium-burn-up and MSR & L1L2H1F2M1 & \\
\hline 11 & BAU+, FR “medium-BR”, high-burn-up and MSR & L1L2H1F3M1 & \\
\hline
\end{tabular}

${ }^{1}$ L1: light water reactor (LWR) with low burn-up ( $45 \mathrm{GWday} / \mathrm{t}$ ); L2: LWR with high burn-up ( $\left.60 \mathrm{GWday} / \mathrm{t}\right)$; H1: heavy water reactor (HWR, typical current prototype); F1: "break-even" fast reactor (FR) with breeding ratio BR 1.0; F2: FR with medium BR (BR 1.2), medium burn-up ( 31 GWday/t); F3: FR with medium BR (BR 1.2), high burn-up ( 54 GWday/t); Al: accelerator driven system (ADS) for minor actinide (MA) burning; M1: molten salt reactor (MSR) for MA burning.

of the GAINS project the following scenarios were selected for the current study (Table 1):

(i) "L1H1": "business-as-usual" (BAU) scenario based on pressurized light water reactors (LWR) (L1) (94\% of power generation) and heavy water reactors (HWR) (H1) $(6 \%)$ operated in a once-through NFC;

(ii) "LIL2H1": the BAU+ scenario based on HWR and LWR replacing high burn-up ALWR (L2) reactors from 2015;

(iii) "L1L2H1F1": scenario with introduction of "breakeven" (BR 1.0) fast reactor (FR) (F1) into BAU+. The FRs are initially introduced starting in 2021. The objective is to have a total generation rate of $10 \mathrm{GW} \cdot \mathrm{a}$ from FRs in 2030 for both growth cases and a total in 2050 of $400 \mathrm{GW} \cdot \mathrm{a}$ for the high case;

(iv) "L1L2H1F2": scenario with introduction of breeder $(\mathrm{BR} \sim 1.2)$ fast reactor $(\mathrm{FR})(\mathrm{F} 2)$ into $\mathrm{BAU}+$;

(v) "L1L2H1F3": scenario with introduction of high burnup breeder (BR 1.2) fast reactor (FR) (F2) into BAU+. The fuel for the high burn-up FR breeder contains MAs and, hence, this reactor contributes to MA burning;

(vi) "L1L2H1F1A1": scenario "L1L2H1F1" with introduction of ADS (A1) which comprises a subcritical fissionable assembly driven by a spallation neutron source. The objective of ADS is transmutation of MAs, and this is how it is employed in the studies of the GAINS. The MA burning in dedicated ADSs eliminates the need for transmutation of MAs in a critical FR, which means that the latter can be optimized for other objectives. In order to reduce the amount of
MAs in the abovementioned scenarios, there is a need to introduce an installed capacity of about $148 \mathrm{GW}$ of ADSs. These represent approximately $3 \%$ of the total installed capacity. The ADSs are only introduced between 2075 and 2100 and only when a look ahead identifies sufficient MAs and plutonium feeds for the 60 year lifetime of the ADS;

(vii) "L1L2H1F2Al"; "L1L2H1F2" scenario with introduction of ADS (A1);

(viii) "L1L2H1F3A1": "L1L2H1F3" scenario with introduction of ADS (A1);

(ix) "L1L2H1F1M1": "L1L2H1F1" scenario with introduction of molten salt reactor (MSR) (M1) for MA burning. In order to reduce the amount of MAs in the abovementioned scenarios, there is a need to introduce an installed capacity of about $160 \mathrm{GW}$ of MSRs. This accounts for around 3\% of total installed capacity;

(x) "L1L2H1F2M1": "L1L2H1F2" scenario with introduction of molten salt reactor (MSR) (M1) for MA burning;

(xi) "L1L2H1F3M1": "L1L2H1F3" scenario with introduction of molten salt reactor (MSR) (M1) for MA burning.

In all scenarios, global nuclear energy generation reaches approximately $1500 \mathrm{GW}$.year by the midcentury and $5000 \mathrm{GW}$.year by 2100 . The GAINS assumptions impose a constraint on the power production by fast reactors in the years between 2030 and 2050 by specifying a maximum deployment rate depending on the overall nuclear energy growth scenario. The objective is to have a total generation 
TABLE 2: Considered key indicators.

\begin{tabular}{lccc}
\hline KI & Abbreviation & Unit & \\
\hline Total natural uranium consumption & KI 1 & ktHM & min \\
Annual SNF generation & KI 2 & ktHM + FP & min \\
Total SNF in long term storages & KI 3 & ktHM + FP & min \\
MA in NFC & KI 4 & tHM & tHM \\
Pu in NFC & KI 5 & ktSWU & min \\
Total enrichment capacities & KI 6 & ktHM + FP & min \\
Total reprocessing capacities & KI 7 & Billion \$ & min \\
Total uranium cost & KI 8 & Billion \$ \\
Total investments in NPPs & KI 9 & & min \\
\hline
\end{tabular}

rate of $10 \mathrm{GW} / \mathrm{a}$ from fast reactors in 2030 and a total of $400 \mathrm{GW} / \mathrm{a}$ in 2050 for the high scenario case. After 2050, deployment rate of fast reactors is maximized and limited only by the amount of plutonium available and the overall nuclear growth rate. Plutonium inventory in the storage was kept close to zero. The calculations for all scenarios were made only by using NFCSS (former VISTA) software [20]. Power production for all selected scenarios is shown in Figure 1 by each reactor type.

3.3. Key Indicators. The following $9 \mathrm{KI}$ were selected and applied (see Table 2). The choice of this set of KI was dictated by the following circumstances: the indicators should be easily evaluated based on data presented in the GAINS project report [2] and the indicators should provide information on the basic problem areas related to the NES deployment (resources, waste management, proliferation resistance, and economics). A short description of the proposed KI is presented below (all of them should be minimized).

(i) "Total natural uranium consumption" (KI 1) is a measure of limited natural uranium resource consumption (a measure of resource sustainability) which represents the total consumed uranium in the timeframe 1970-2100 by all types of nuclear reactors.

(ii) "Annual SNF generation" (KI 2) serves as a measure for SNF management efficiency and presents annual SNF discharge shipped to at-reactor storages in 2100 from all types of nuclear reactors.

(iii) “Total SNF in long term storages" (KI 3) presents a possible measure for SNF management efficiency and corresponds to integral SNF amount from all types of nuclear reactors accumulated in away-from-reactor storage facilities by 2100 .

(iv) "MA in NFC" (KI 4) is a measure for nuclear waste management efficiency which represents total MA inventories at all NFC steps produced by each nuclear reactor type in 2100 .

(v) "Pu in NFC" (KI 5) provides a measure of proliferation resistance and presents the total plutonium amount produced by each nuclear reactor type which was circulated and accumulated at all NFC steps in 2100 . (vi) “Total enrichment capacities” (KI 6) serves as another proliferation resistance measure and identifies cumulative requirements in the timeframe 2008-2100 in uranium enrichment to produce nuclear fuel for each nuclear reactor.

(vii) "Total reprocessing capacities" (KI 7) is a cumulative reprocessing capacity requirement to reprocess SNF from each nuclear reactor in the timeframe 20082100 and specifies one more proliferation resistance measure.

(viii) "Total uranium cost" (KI 8) is another measure of the economic efficiency of limited natural uranium resource consumption (a possible measure of resources sustainability). KI 8 represents the total uranium recovery cost needed to produce nuclear fuel for deployment scenarios by 2100 . This KI was estimated based on the following: (1) the assessment was carried out for the lower estimation of uranium recovery cost according to [21]; (2) in all considered scenarios all uranium reserves with the estimated recovery cost were consumed (13,800 kt); (3) the remainder of uranium reserves (the majority of consumed uranium reserves) was recovered by $\$ 260$ per kg; (4) the discount rate was chosen to be $5 \%$.

(ix) "Total investments in NPPs" (KI 9) presents a possible measure of economic efficiency of nuclear power development and characterizes the total discounted investment cost in NPP construction till 2100. To assess the investments required for the deployment of nuclear reactors according to the considered scenarios, the following assumptions were made: (1) the overnight capital construction costs of existing nuclear reactor types (LWR and HWR) were supposed to be equal to $4000 \$ / \mathrm{kWe}$; (2) for the construction of new types of nuclear reactors (ALWR, FR1, FR2, FR3, ADS, MSR) the overnight capital costs were taken more than those of existing nuclear reactor types by $10 \%, 20 \%, 25 \%, 30 \%, 40 \%$, and $40 \%$, respectively; (3) The discount rate was chosen to be $5 \%$.

The performance table (Table 3) containing evaluated KI values for all considered NESs was prepared based on formulated assumptions and the data presented in the Excel 
L1H1 and L1L2H1
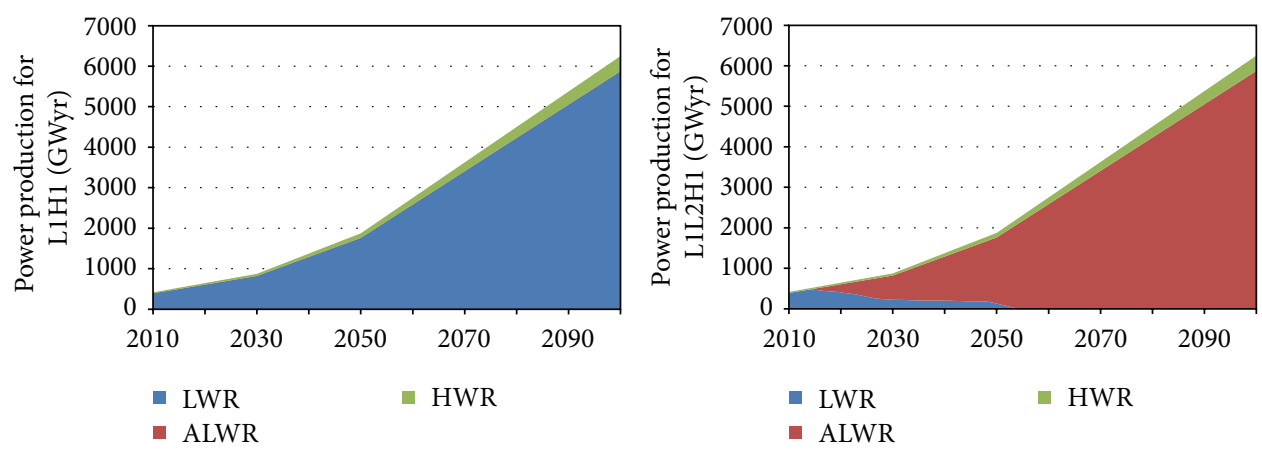

L1L2H1F1, L1L2H1F2, and L1L2H1F3
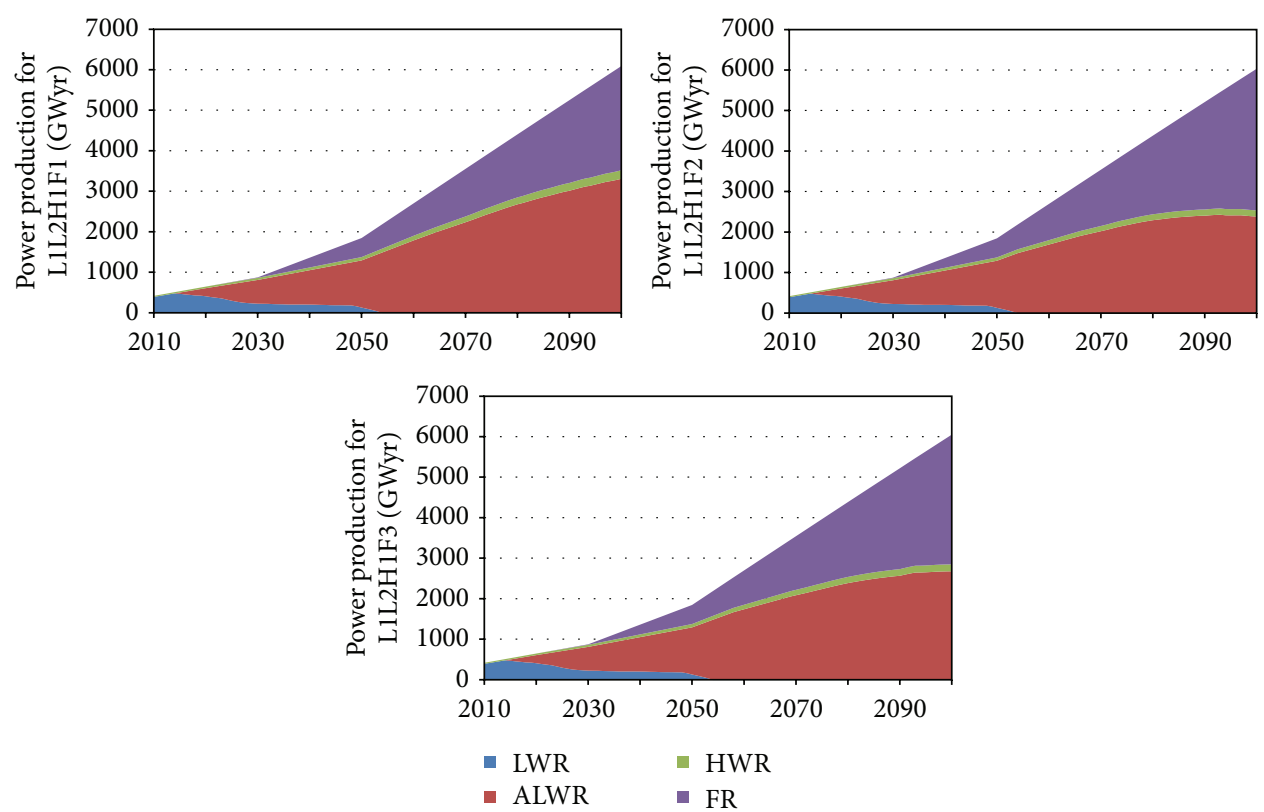

L1L2H1F1A1, L1L2H1F2A1, and L1L2H1F3A1

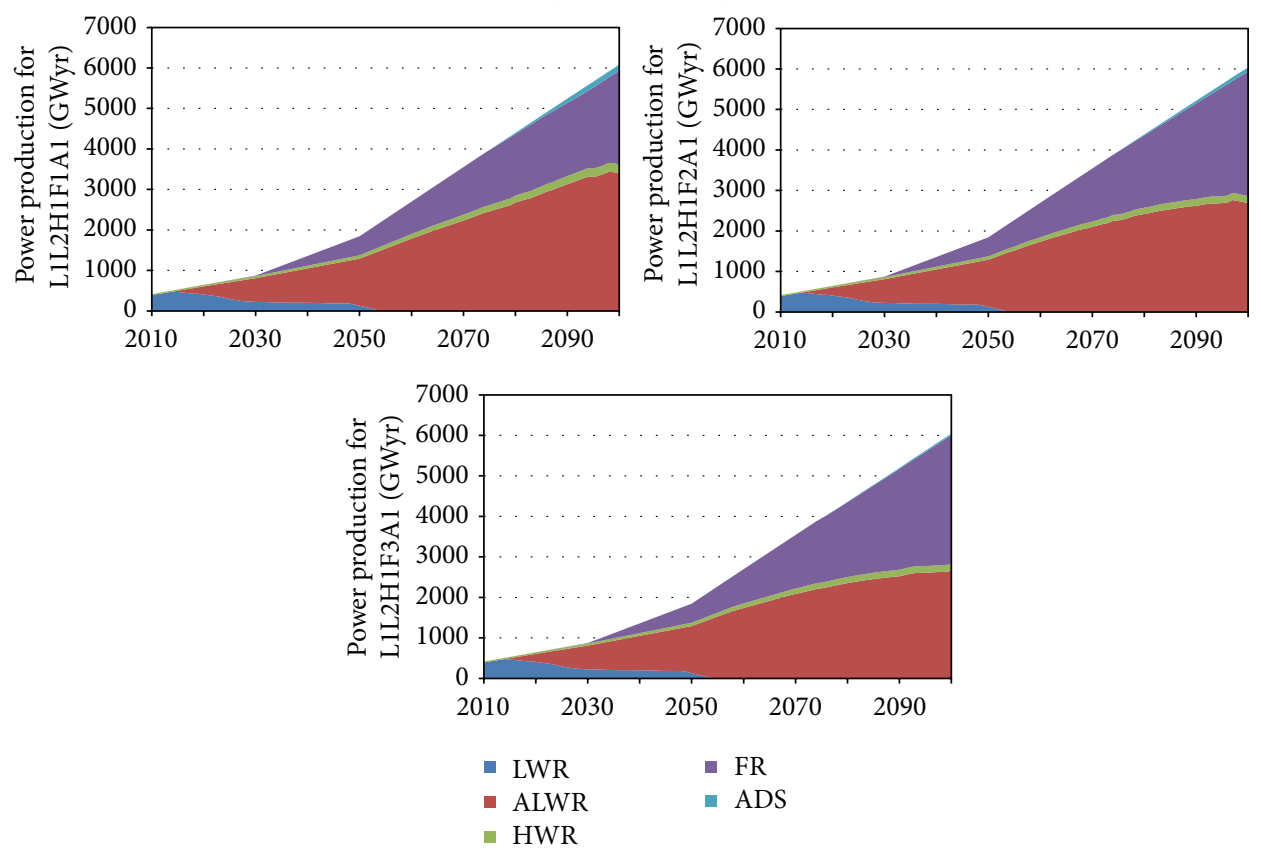

(a)

FIgure 1: Continued. 


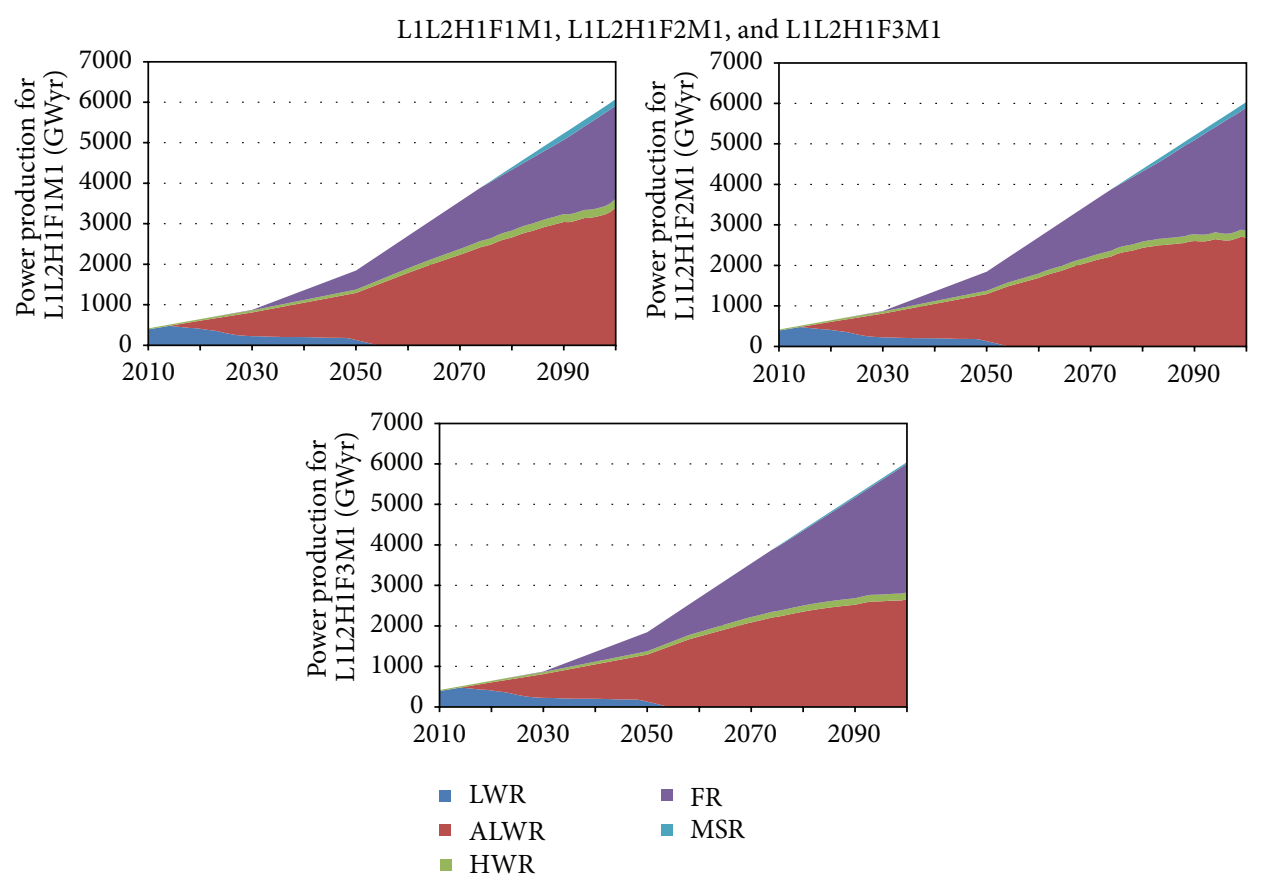

(b)

FIGURE 1: Power production by NESs in considered NES evolution scenarios.

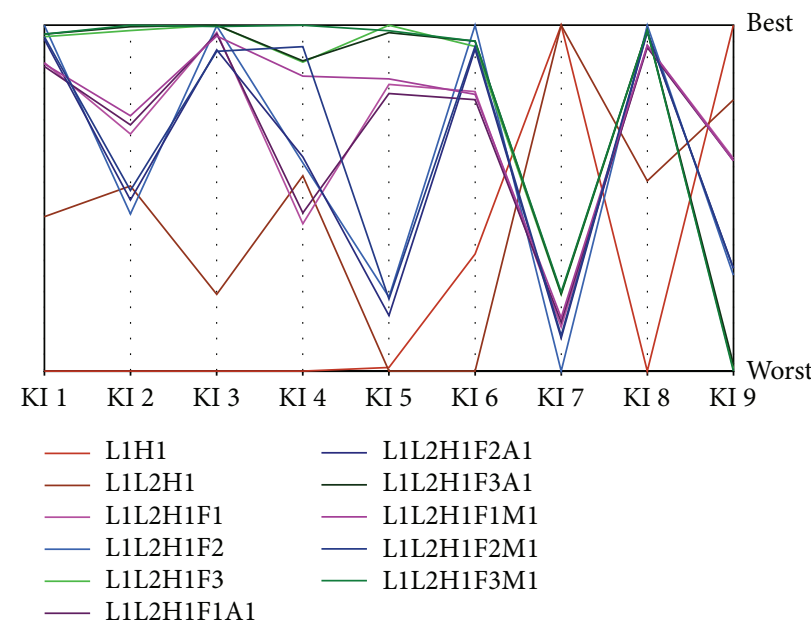

(a)

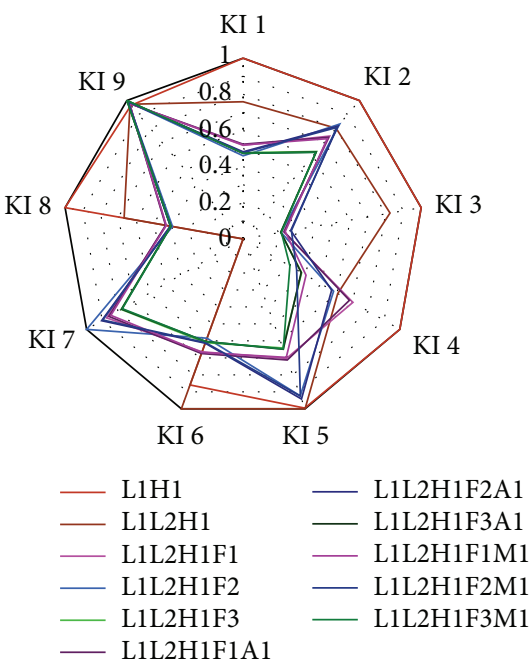

(b)

FIGURE 2: Value path (a) and radar chart (b).

tables of supplementary materials for the GAINS collaborative project report [2]. Visualization of the performance table may be presented in the form of the so-called value path and radar chart (Figure 2). The value paths diagram shows variations in the values of all KI for the entire set of NESs and allows evaluating quantitatively in relative terms how much improvement in the value of one KI deteriorates the values of other KI due to the transition from one NES to another. A radar chart (also known as web chart, spider chart, etc.) is a graphical method of displaying multivariate data in the form of a two-dimensional chart of three or more quantitative variables represented on axes starting from the same point.

In the most evident form the procedure of multicriteria assessment of INESs may be formulated as an identification of the closeness of each INES by a set of KI to the desired area, the upper limit of graph (value paths) or the diagram center (radar chart). Obviously, the closeness will depend on the metric type which serves a measure of distance to the desired area, but in this regard to make definite judgments on this matter is impossible. 


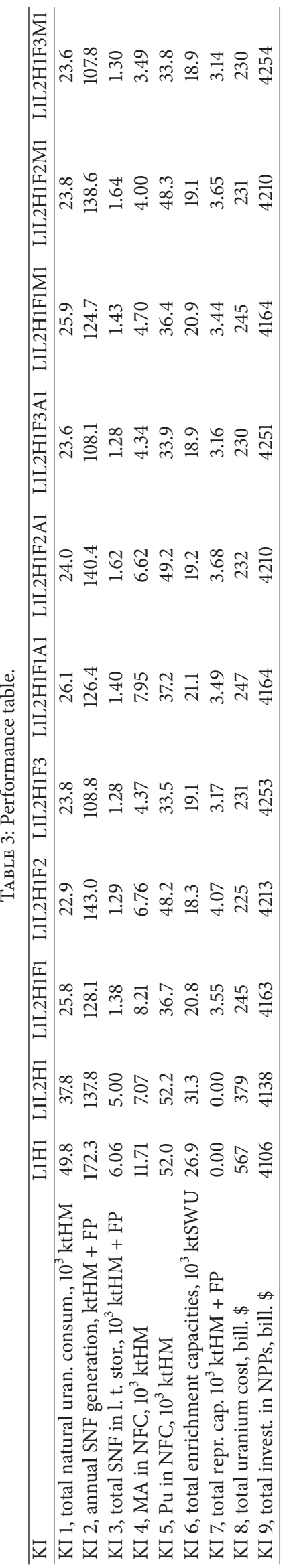


TABLE 4: Weights' summary table.

\begin{tabular}{|c|c|c|c|c|c|}
\hline \multirow{2}{*}{ KI } & \multicolumn{5}{|c|}{ Variants } \\
\hline & 1 & $2 \mathrm{a}$ & $2 b$ & 3 & 4 \\
\hline Total natural uranium consumption & 0,11 & 0,11 & 0,12 & 0,03 & 0,02 \\
\hline Annual SNF generation & 0,11 & 0,13 & 0,12 & 0,03 & 0,6 \\
\hline Total SNF in long term storages & 0,11 & 0,13 & 0,12 & 0,03 & 0,18 \\
\hline MA in NFC & 0,11 & 0,10 & 0,11 & 0,03 & 0,1 \\
\hline $\mathrm{Pu}$ in NFC & 0,11 & 0,10 & 0,11 & 0,03 & 0,02 \\
\hline Total enrichment capacities & 0,11 & 0,10 & 0,10 & 0,03 & 0,02 \\
\hline Total reprocessing capacities & 0,11 & 0,10 & 0,10 & 0,03 & 0,02 \\
\hline Total uranium cost & 0,11 & 0,13 & 0,10 & 0,11 & 0,02 \\
\hline Total investments in NPPs & 0,11 & 0,11 & 0,12 & 0,68 & 0,02 \\
\hline
\end{tabular}

Let us emphasize an issue associated with a set of nondominated scenarios. The determination of a set of nondominated scenarios and the information about domination may be used to exclude dominated alternatives from the consideration. The nondominated (e.g., Pareto optimal, Pareto efficient, or noninferior, etc.) solution is a solution for which none of the KI can be improved in value without degrading some of the other objective values. Without additional subjective preference information all nondominated solutions are considered equally good (compromised, tradeoff). In the considered case all alternatives are nondominated. It could be said that each alternative has some advantages over others.

3.4. Weighting Factors. The identification of weights (formalization of the experts' preferences) is the most sensitive issue in formal application of the MCDA methods; it requires accuracy and reasonableness. The following options were considered reflecting possible experts' preferences regarding desirable goals that NES evolution scenarios should achieve:

(i) equal significance of all KI (variant number 1),

(ii) expert preferences based on questionnaires distributed at the INPRO meetings (Two groups of experts: expert group (a) INPRO Dialogue Forum4 participants and expert group (b) SYNERGIES participants, correspondingly [22].) (variant number $2 \mathrm{a}$ and variant number $2 \mathrm{~b}$ ),

(iii) preference to investments minimization (variant number 3),

(iv) preference to wastes minimization (variant number 4).

In the analysis the same weights for all methods were used. Within the framework of multicriteria analysis this requirement is not mandatory but seems to be very strict. The final weight values (Table 4) were discussed with and agreed by subject matter experts.

3.5. Ranking Results and Sensitivity Analysis. All the alternatives (NES evolution scenarios) can be categorized in three groups: the first group includes the scenarios with F3 type fast reactor, the second group consists of the scenarios with
F2 and F1 type fast reactor, and the third group contains the scenarios based on once-through NFC (Figure 3). To combine the alternatives in these groups makes sense because the general indicator values in each applied MCDA method are close for the corresponding scenario families. Such classification provides a clearer interpretation of the ranking results. Figure 3 shows ranking results for the MAVT method with weight variant number 1 .

The applied MCDA methods and simple scoring model have shown that, despite some differences in the ranking alternatives, the results obtained by using different methods are well coordinated and consistent, which allows to differentiate the attractiveness of different NES evolution scenarios and to make a number of general conclusions regarding the effectiveness of relevant reactor technologies in the considered NESs (Table 5).

The ranking results demonstrate that the NES evolution scenarios based on F3 type fast reactor are the more attractive options and the scenarios based on once-through NFC are the least attractive ones. The scenarios based on F1 and F2 type fast reactor have shown some variation in ranks that does not allow providing a definitive judgment regarding the attractiveness of the corresponding NESs. The same is true for MA burners (MSR and ADS).

For problem considered, all of the applied MCDA methods (MAVT, MAUT, PROMETHEE, TOPSIS, AHP, and SSM used for variants numbers 1,3 , and 4) demonstrate the superiority of the first and second groups of alternatives over the third one (Table 6). The ranking results for variants numbers $1,2 \mathrm{a}$, and $2 \mathrm{~b}$ obtained by using any methods are the same and correlate significantly with the results for variant number 4 . The ranking result for weights' variant number 3 is considerably different in comparison with other variants (Table 6).

Analysis of the numerical example based on abovementioned methods has shown that five methods (MAVT, MAUT, PROMETHEE, AHP, and SSM) give similar ranking results for all variants, while the TOPSIS method generally gives similar results too, but not for variant number 3 (because of the specifics of ranking algorithm realized in TOPSIS).

The MCDA methods can be used to support evaluation of NES for a more distant future, as provided for by the objective of the KIND project, but MCDA is only a means. 
TABLE 5: Comparative analysis of ranking results by different methods (for weight variant number 1).

\begin{tabular}{|c|c|c|c|c|c|c|}
\hline Rank & SSM & MAVT & MAUT & TOPSIS & PROMETHEE & AHP \\
\hline 1 & L1L2H1F3A1 & L1L2H1F3M1 & L1L2H1F3M1 & L1L2H1F3M1 & L1L2H1F3M1 & L1L2H1F3M1 \\
\hline 2 & L1L2H1F3M1 & L1L2H1F3A1 & L1L2H1F3A1 & L1L2H1F3A1 & L1L2H1F3A1 & L1L2H1F3 \\
\hline 3 & L1L2H1F3 & L1L2H1F3 & L1L2H1F3 & L1L2H1F3 & L1L2H1F3 & L1L2H1F3A1 \\
\hline 4 & L1L2H1F2 & L1L2H1F1M1 & L1L2H1F1M1 & L1L2H1F1M1 & L1L2H1F1M1 & L1L2H1F1M1 \\
\hline 5 & L1L2H1F1M1 & L1L2H1F1A1 & L1L2H1F1A1 & L1L2H1F2M1 & L1L2H1F1A1 & L1L2H1F2M1 \\
\hline 6 & L1L2H1F1 & L1L2H1F1 & L1L2H1F1 & L1L2H1F1A1 & L1L2H1F1 & L1L2H1F2 \\
\hline 7 & L1L2H1F2M1 & L1L2H1F2M1 & L1L2H1F2M1 & L1L2H1F2A1 & L1L2H1F2M1 & L1L2H1F1 \\
\hline 8 & L1L2H1F1A1 & L1L2H1F2 & L1L2H1F2 & L1L2H1F1 & L1L2H1F2A1 & L1L2H1F1A1 \\
\hline 9 & L1L2H1F2A1 & L1L2H1F2A1 & L1L2H1F2A1 & L1L2H1F2 & L1L2H1F2 & L1L2H1F2A1 \\
\hline 10 & L1L2H1 & L1L2H1 & L1L2H1 & L1L2H1 & L1L2H1 & L1L2H1 \\
\hline 11 & L1H1 & L1H1 & L1H1 & L1H1 & L1H1 & L1H1 \\
\hline
\end{tabular}

TABLE 6: Ranking of the NES evolution scenarios.

\begin{tabular}{|c|c|c|c|c|c|c|c|}
\hline Variants & The best NESs & & $\rightarrow$ & & $\rightarrow$ & & The worst NESs \\
\hline Numbers $1,2 \mathrm{a}, 2 \mathrm{~b}$, and 4 & $\begin{array}{c}\text { L1L2H1F3 } \\
\text { L1L2H1F3A1 } \\
\text { L1L2H1F3M1 }\end{array}$ & $>$ & $\begin{array}{c}\text { L1L2H1F1 } \\
\text { L1L2H1F2 } \\
\text { L1L2H1F1A1 } \\
\text { L1L2H1F2A1 } \\
\text { L1L2H1F1M1 } \\
\text { L1L2H1F2M1 }\end{array}$ & $>$ & L1L2H1 & $>$ & L1H1 \\
\hline Number 3 & $\begin{array}{c}\text { L1H1 } \\
\text { L1L2H1 }\end{array}$ & $>$ & $\begin{array}{c}\text { L1L2H1F1 } \\
\text { L1L2H1F1A1 } \\
\text { L1L2H1F1M1 }\end{array}$ & $>$ & $\begin{array}{c}\text { L1L2H1F2 } \\
\text { L1L2H1F2A1 } \\
\text { L1L2H1F2M1 }\end{array}$ & $>$ & $\begin{array}{c}\text { L1L2H1F3 } \\
\text { L1L2H1F3A1 } \\
\text { L1L2H1F3M1 }\end{array}$ \\
\hline
\end{tabular}

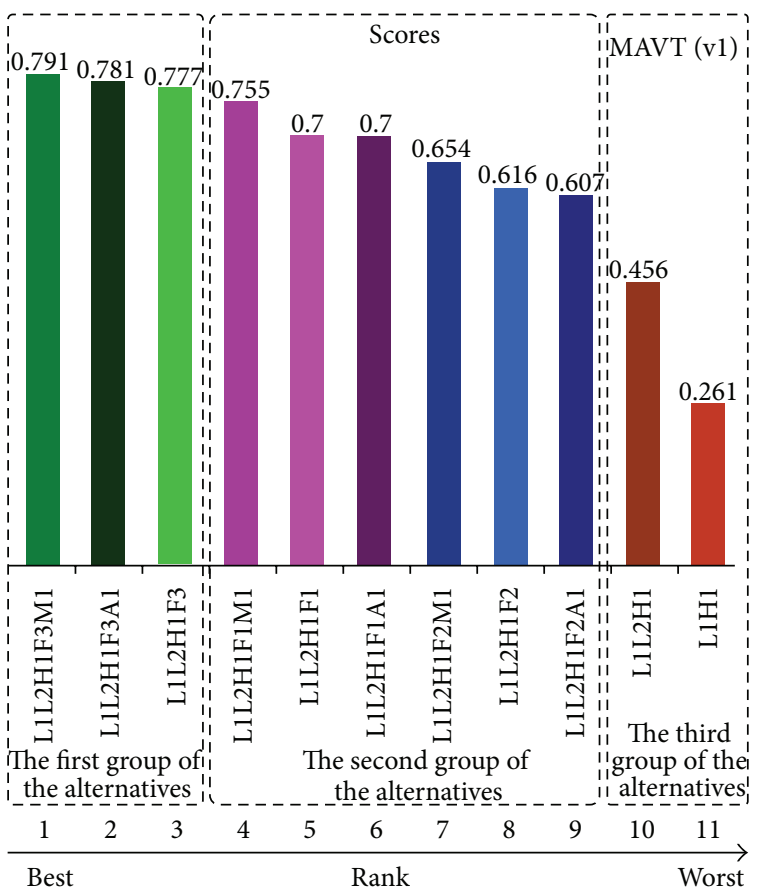

FIGURE 3: Ranking results for the MAVT method with weight variant number 1 .
The more important output to support decision makers is the identification (in a clear and understandable manner) of the features of a NES that results in the risks and benefits to be identified. Table 7 illustrates, by means of "heat map (A heat map is a graphical representation of data where different values contained in the matrix are represented by different colours.)" technique, the ranking of the NES evolution scenarios by individual KI, which provides highlighting merits and demerits associated with the considered NES evolution scenarios.

One of the possible options for interpretation of the MAVT ranking results is a decomposition of multiattribute value functions on the individual components. Depending on the depth and level of details, the interpretation needs to be done and this decomposition may be performed in different ways. In Figure 4 the values of the high-level objectives titled "costs" and "risks" (both of them should be kept as low as possible) for the considered alternatives are presented in the form of two-dimensional scatter graph. This graph clearly illustrates high-level performance efficiency associated with each NES evolution scenario and provides opportunity to identify high-level merits and demerits related to the considered options. "Costs" and "risks" high-level objectives were evaluated based on single-attribute value functions for KI number 1, 8, and 9 and for KI numbers 2, 3, 


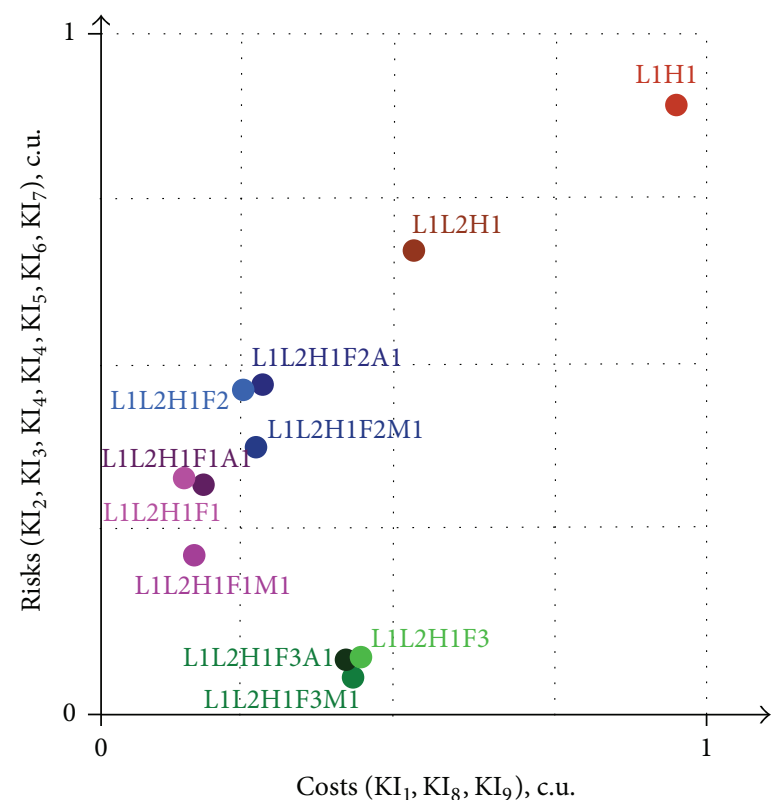

Figure 4: Values of the high-level objectives.

$4,5,6$, and 7, correspondingly. In that case, if decision maker and experts want further explanation of the reasons that have led to the dominance of one alternative over another, the multiattribute value function should be decomposed into components corresponding to each assessment area.

In the study, uncertainties were examined through a sensitivity analysis. This analysis assumed sensitivity to weights and value, utility and preference functions changing. The sensitivity analysis confirmed that the ranking results are generally stable to possible changes in model assumptions both, as comes to the methods' parameters and the weights' values. Based on results weights' sensitivity analysis it may be concluded that the ranking results are not extremely sensitive to the values of the weighting factors and may be considered stable ones because they satisfy the standard formal sensitivity test: the change of all weighting factors on $10 \%$ does not change the ranks of alternatives. The approach implemented in this study (using a wide range of essentially different MCDA methods) has demonstrated the closeness of the results obtained by different methods and confirmed that the ranking results are robust and stable.

3.6. Discussions. Taking into account the results of the sensitivity analysis, the additional analysis of alternatives by the supplementary methods, and the entire set of graphical and attribute information, the best alternatives could be identified in different situations. The recommendations regarding preferences in the considered reactor technologies and the corresponding NES evolution scenarios may be provided based on the analysis performed for different circumstances.

If the requirement to minimize investment costs will not be determinative and restrictive, the priority could be given to one of the scenarios with F3 type fast reactors ("L1L2H1F3," "L1L2H1F3A1," and "L1L2H1F3M1"). The most efficient material flow management (reduction of waste generation, proliferation risks, etc.) is provided by "L1L2H1F3M1" which is by $0.7 \%$ more costly (relative increase in the values of KI 9) compared to "L1L2H1F3."

If the requirement to minimize investment costs will be dominant, the "L1L2H1" scenario seems to be a better alternative compared to "L1H1." The "L1L2H1" scenario will provide greater system efficiency without significantly increasing the investment costs $(0.8 \%)$ in comparison with the cheapest "L1H1" option.

The "L1L2H1F1" scenario is a trade-off or compromise. This alternative provides increase in the investment cost by $1.4 \%$ in comparison with the cheapest " $\mathrm{L} 1 \mathrm{H1}$ " scenario and, at the same time, offers acceptable performance efficiency for nuclear materials management.

Talking about related nuclear technologies it should be noted that, from the viewpoint of the complete NES, F2 type fast reactor is less attractive than its competitors: F3 type fast reactor, which is more expensive but more efficient for the system in terms of NFC material flow management, and F1 type fast reactor, which is cheaper but provides system efficiency comparable to F2 type fast reactor.

Notwithstanding that the MSR seems more attractive in comparison with the ADS, it should be admitted that there is no meaningful difference between the MSR and ADS in the considered NES evolution scenarios because of their small share in the systems. As a result, to make a choice between these alternatives it would be necessary to carry out further analysis assuming their increasing shares in the structures of the corresponding NESs.

Application of the considered methods to multicriteria assessment of the efficiency of various NES evolution scenarios shows that, despite some differences in the ranking alternatives, the results obtained by using different methods are well coordinated and consistent. In general, it may be expected that, in comparable conditions, the results obtained by using different methods will be well harmonized and steady. Obviously, a set of relevant methods should not be limited to just the ones considered in this study. Experts may express their preference for other ranking/aggregation methods, but verification regarding the use of the corresponding methods for a specific problem solution should be provided. It is also reasonable to use the approach applied in this study when several different ranking/aggregation methods are being used. Such an approach may facilitate more deep understanding and better analysis the problem, providing an additional sensitivity analysis of the obtained ranking results which would increase confidence level of the study.

Recommendations for the KIND project regarding advanced methods of expert judgment aggregation are as follows. In general, all of the considered MCDA methods including MAVT, MAUT, TOPSIS, PROMETHEE, and AHP can be used for multicriteria comparison of NESs both technology and scenario based. It is also recommended to make a final choice of the most appropriate method for a particular problem on the basis of the analysis of problem context and initial information quality provided by subject matter experts.

For the objective of the KIND project, the use of simple methods could be recommended, given the application to less 


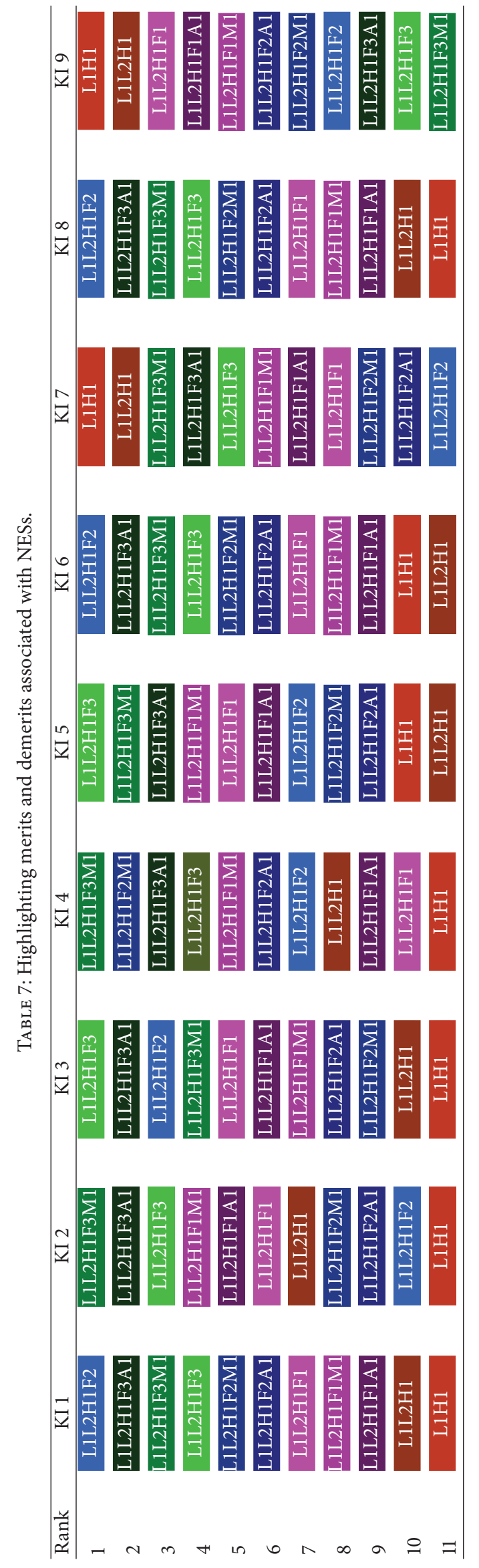


mature technologies. In other words, simple additive weighting and MAVT methods could be used and examination of uncertainties through a sensitivity analysis or uncertainty calculations should be provided.

Representation of preferences among different criteria (identification of weights) is the most sensitive issue in formal application of the MCDA methods that requires accuracy and reasonableness. Weighting of indicators or areas can be done based on national preferences and standpoints on nuclear energy development. Development of single-attribute value functions is also a potentially sensitive area that might be confusing to users. A clear explanation of these functions and their elaboration procedure should be included in the methodology being developed by the KIND project.

While MCDA methods could be recommended for use in support of NES comparative evaluation for a more distant future, MCDA is still only a means. The more important output to support decision makers is the identification (in a clear and understandable manner) of the features of a NES (or NES evolution scenario) that result in the benefits and risks.

\section{Conclusions}

The prospects of nuclear power to meet future energy needs are vast. However, it is extremely difficult to achieve a common view of an optimal pathway for its deployment. The contradictory nature may be traced in every nuclear engineering field, once one is looking for certain optimal technological and institutional solutions at different levels. Lack of common methodologies for decision making in multicriteria formulation for nuclear reactors, related NFC, NES, and NES evolution scenario efficiency assessment and, in particular, in the area of the NES sustainability assessments complicates the procedure of formulating a coordinated vision of preferable technological and institutional solutions, balanced on different benefits and risks common to several countries or specific for a particular country.

Development and application of state-of-the-art MCDM tools for comparative NES or evolution scenario assessment and optimization appears necessary because of the growing need for highlighting conflicts and finding compromises in the decision making process. The example presented in this study shows that application of such methods may provide added value to the analysis of possible options and yield a reasonable stable, well-interpreted, and decision making oriented results clarifying the pros and cons of the considered alternatives on quantitatively and methodologically proven and well-elaborated foundations. The results of the illustrative numerical example added a degree of clarity (better understanding) with respect to the GAINS framework application for ranking of options based on a set of KI using a set of preferences.

The presented example is one of the case studies being analyzed in the framework of the new INPRO Collaborative Project on Key Indicators for Innovative Nuclear Energy Systems, which is oriented to supporting practical decision making and representing a functional guidance and tool for the support of decision making process in the nuclear reactors, related nuclear fuel cycles, and nuclear energy systems areas.

\section{Abbreviations}

\begin{tabular}{|c|c|}
\hline ADS: & Accelerator driven system \\
\hline AHP: & Analytic Hierarchy Process \\
\hline ALWR: & Advanced light water reactor \\
\hline BAU: & Business-as-usual \\
\hline BR: & Breeding ratio \\
\hline BWR: & Boiling water reactor \\
\hline FP: & Fission product \\
\hline FR: & Fast reactor \\
\hline GAINS: & $\begin{array}{l}\text { Global Architecture of Innovative Nuclear } \\
\text { Energy Systems Based on Thermal and } \\
\text { Fast Reactors Including a Closed Fuel } \\
\text { Cycle }\end{array}$ \\
\hline HM: & Heavy metal \\
\hline HWR: & Heavy water reactor \\
\hline IAEA: & International Atomic Energy Agency \\
\hline INPRO: & $\begin{array}{l}\text { International Project on Innovative } \\
\text { Nuclear Reactors and Fuel Cycles }\end{array}$ \\
\hline INES: & Innovative nuclear energy system \\
\hline NES: & Nuclear energy system \\
\hline NFC: & Nuclear fuel cycle \\
\hline NFCSS: & Nuclear fuel cycle simulation system \\
\hline KI: & Key indicator \\
\hline KIND: & $\begin{array}{l}\text { Key Indicators for Innovative Nuclear } \\
\text { Energy Systems }\end{array}$ \\
\hline LWR: & Light water reactor \\
\hline MA: & Minor actinide \\
\hline MAVT: & Multiattribute Value Theory \\
\hline MAUT: & Multiattribute Utility Theory \\
\hline MCDA: & Multicriteria Decision Analysis \\
\hline MCDM: & Multiple-Criteria Decision Making \\
\hline MODM: & Multiobjective Decision Making \\
\hline MSR: & Molten salt reactor \\
\hline NPP: & Nuclear power plant \\
\hline PROMETHEE: & $\begin{array}{l}\text { Preference Ranking Organization Method } \\
\text { for EnrichmentEvaluations }\end{array}$ \\
\hline Pu: & Plutonium \\
\hline SNF: & Spent nuclear fuel \\
\hline SSM: & Simple scoring model \\
\hline SWU: & Separative work unit \\
\hline TOPSIS: & $\begin{array}{l}\text { Technique for Order Preference by } \\
\text { Similarity to the Ideal Solution. }\end{array}$ \\
\hline
\end{tabular}

\section{Conflict of Interests}

The authors declare that there is no conflict of interests regarding the publication of this paper.

\section{References}

[1] International Atomic Energy Agency, "International Project on Innovative Nuclear Reactors and Fuel Cycles (INPRO)," http://www.iaea.org/INPRO.

[2] International Atomic Energy Agency, Framework for Assessing Dynamic Nuclear Energy Systems for Sustainability, Final Report of the INPRO Collaborative Project on Global Architectures of Innovative Nuclear Energy Systems with Thermal and Fast Reactors and a Closed Nuclear Fuel Cycle (GAINS), IAEA 
Nuclear Energy Series NP-T-1.14, 2013, http://www-pub.iaea .org/books/IAEABooks/8873/Framework-for-AssessingDynamic-Nuclear-Energy-Systems-for-Sustainability-FinalReport-of-the-INPRO-Collaborative-Project-GAINS.

[3] A. Andrianov, I. Kuptsov, and V. Murogov, "Towards sustainable nuclear power development," International Journal for Nuclear Power, vol. 59, no. 5, pp. 287-293, 2014.

[4] A. A. Andrianov, "Approaches and software for multi-objective optimization of nuclear power structures," Sustainability, vol. 4, no. 4, pp. 721-739, 2012.

[5] J. Figueira, G. Salvatore, and E. Matthias, Multiple Criteria Decision Analysis: State of the Art Surveys, Springer Science; Business Media, Boston, Mass, USA, 2005.

[6] V. Belton and T. Stewart, Multiple Criteria Decision Analysis: An Integrated Approach, Kluwer Academic, Dordrecht, The Netherlands, 2002.

[7] R. Keeney and H. Raiffa, Decision with Multiple Objectives, John Wiley \& Sons, New York, NY, USA, 1976.

[8] D. von Winterfeldt and W. Edwards, Decision Analysis and Behavioral Research, Cambridge University Press, Cambridge, UK, 1986.

[9] C.-L. Hwang and K. Yoon, Multiple Attribute Decision Making: Methods and Applications, Springer, Berlin, Germany, 1981.

[10] J.-P. Brans and P. Vincke, "A preference ranking organisation method: (The PROMETHEE method for multiple criteria decision-making)," Management Science, vol. 31, no. 6, pp. 647656, 1985.

[11] T. L. Saaty, The Analytic Hierarchy Process, McGraw-Hill, New York, NY, USA, 1980.

[12] R. Keeney and K. Nair, Evaluating Potential Nuclear Power Plant Sites in the Pacific Northwest Using Decision Analysis, International Institutr for Applied System Analysis, Laxenburg, Austria, 1976.

[13] “The Nirex case study (Generic Repository Studies) 1988-1990”.

[14] J. S. Dyer, J. C. Butler, T. Edmunds, and J. Jia, "A multiattribute utility analysis of alternatives for the disposition of surplus weapons-grade plutonium," Operations Research, vol. 46, no. 6, pp. 749-762, 1998.

[15] R. P. Hämäläinen, M. R. K. Lindstedt, and K. Sinkko, "Multiattribute risk analysis in nuclear emergency management," Risk Analysis, vol. 20, no. 4, pp. 455-467, 2000.

[16] J. C. Butler, A. N. Chebeskov, J. S. Dyer, T. A. Edmunds, J. Jia, and V. I. Oussanov, "The United States and Russia evaluate plutonium disposition options with multiattribute utility theory," Interfaces, vol. 35, no. 1, pp. 88-101, 2005.

[17] W. S. Charlton, R. F. Lebouf, C. Gariazzo et al., "Proliferation resistance assessment methodology for nuclear fuel cycles," Nuclear Technology, vol. 157, no. 2, pp. 143-156, 2007.

[18] S. K. Kim, H. S. Park, and C. H. Jung, "MAUT approach for selecting a proper decommissioning scenario," in Proceedings of the (WM '07) Conference, Tucson, Ariz, USA, February-March 2007.

[19] W. Schenler, P. Burgherr, C. Bauer, and S. Hirschberg, "PSI/ NEEDS project," 2009, http://www.needs-project.org/.

[20] IAEA-TECDOC-1535, "Nuclear Fuel Cycle Simulation System (VISTA)".

[21] Uranium 2011 Resources, Production and Demand OECD, 2012, International Atomic Energy Agency.

[22] International Atomic Energy Agency; 4th INPRO Dialogue Forum, Drivers and Impediments for Regional Cooperation on the Way to Sustainable Nuclear Energy Systems, International Atomic Energy Agency, 4th INPRO Dialogue Forum, Vienna, Austria, 2012, http://www.iaea.org/INPRO/DFs/index.html. 


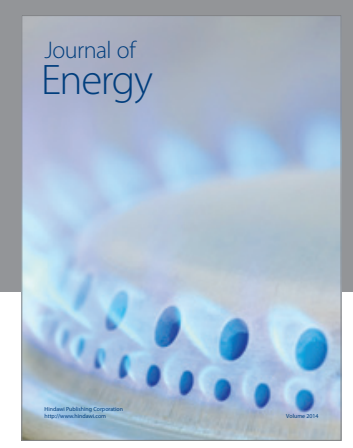

Journal of

Industrial Engineering
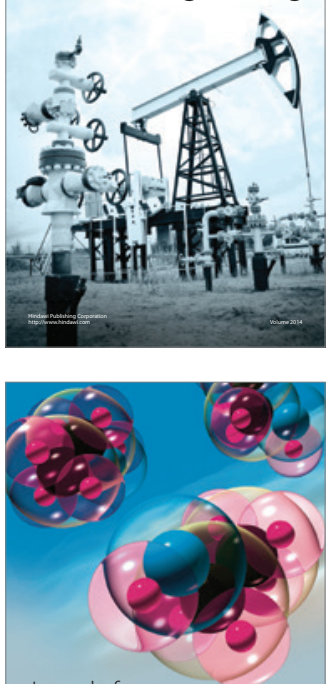

Fuels
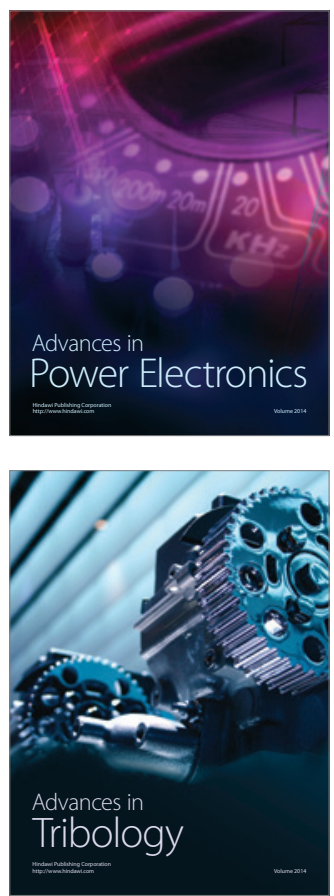

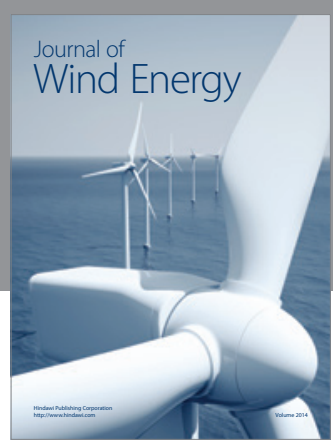

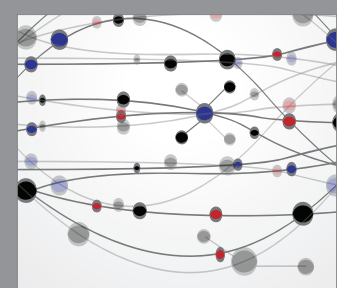

The Scientific World Journal

Submit your manuscripts at http://www.hindawi.com

Journal of

Structures
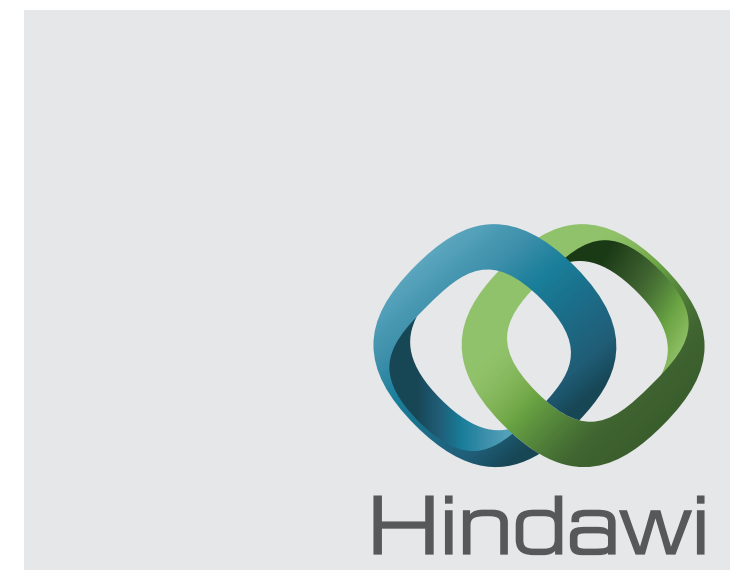

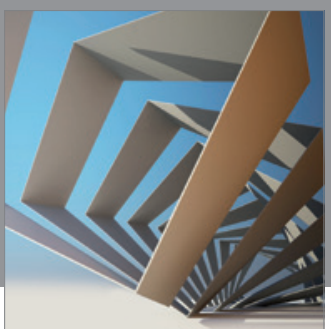

Rotating

Machinery
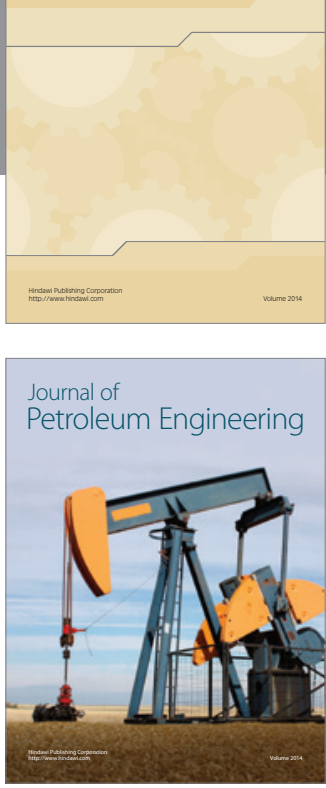

Journal of

Solar Energy
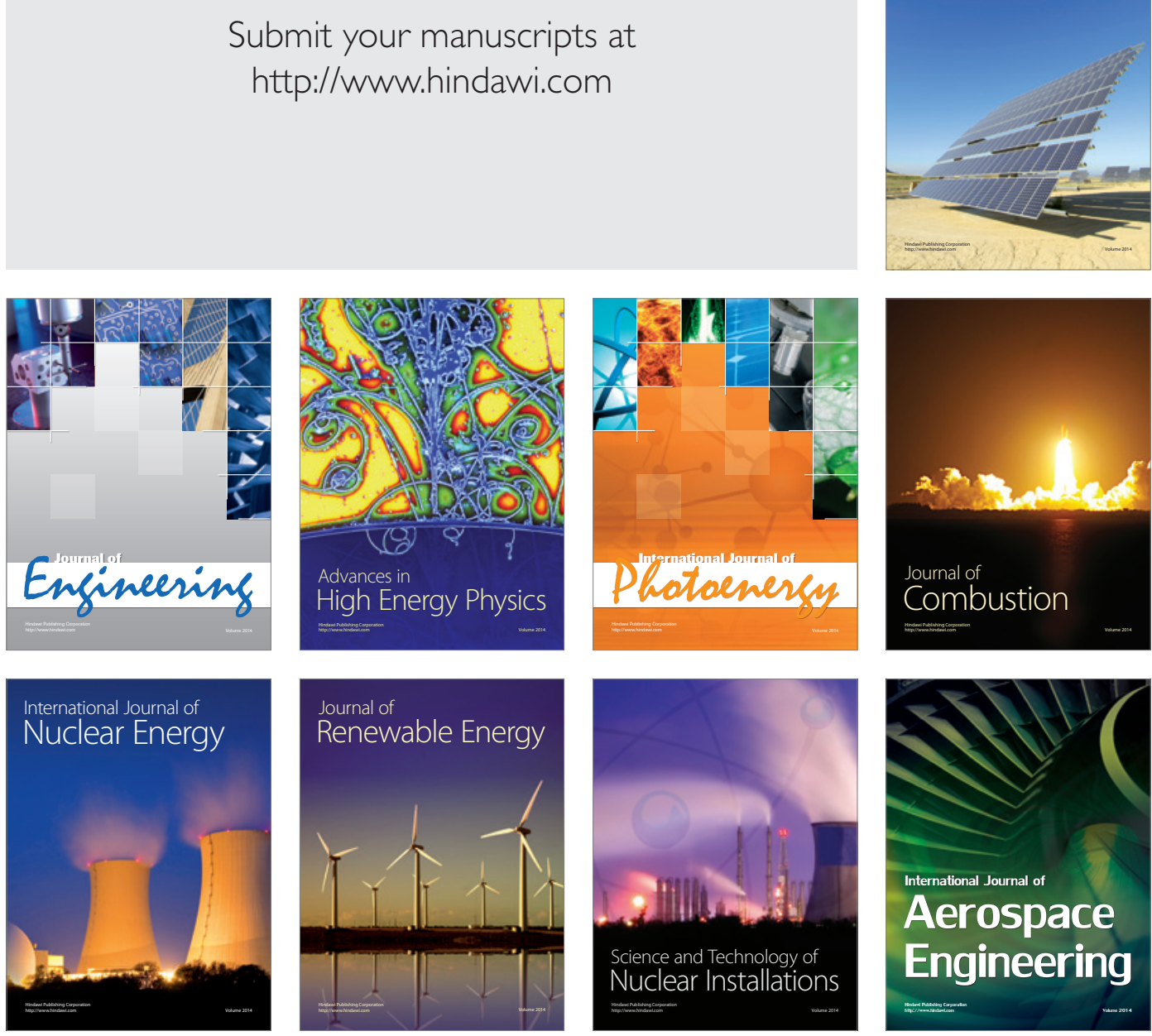